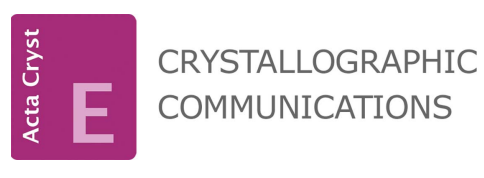

ISSN 2056-9890

Received 27 October 2017

Accepted 9 January 2018

Edited by A. M. Chippindale, University of Reading, England

Keywords: crystal structure; zinc thiosulfate; piperazine; hydrogen bonding.

CCDC reference: 1571150

Supporting information: this article has supporting information at journals.iucr.org/e

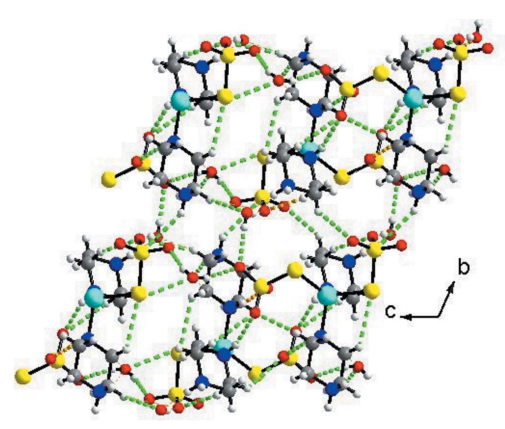

OPEN $\odot$ ACCESS

\section{Crystal structure of bis(piperazin-1-ium- $\left.\kappa N^{4}\right)$ - bis(thiosulfato- $\kappa S$ )zinc(II) dihydrate}

\author{
Avijit Kumar Paul* \\ Department of Chemistry, National Institute of Technology Kurukshetra, Haryana 136119 , India. *Correspondence \\ e-mail: apaul@nitkkr.ac.in
}

In the title compound, $\left[\mathrm{Zn}\left(\mathrm{C}_{4} \mathrm{H}_{11} \mathrm{~N}_{2}\right)_{2}\left(\mathrm{~S}_{2} \mathrm{O}_{3}\right)_{2}\right] \cdot 2 \mathrm{H}_{2} \mathrm{O}$, two thiosulfate ions coordinate to the zinc(II) atom through the terminal $\mathrm{S}$ atoms. The tetrahedral coordination around the $\mathrm{Zn}^{\mathrm{II}}$ ion is completed by ligating to two $\mathrm{N}$ atoms of two piperazinium ions. The remaining two $\mathrm{N}$ atoms of the piperazinium ions are diprotonated and do not coordinate to the metal centre. In the crystal, however, they are involved in $\mathrm{N}-\mathrm{H} \cdots \mathrm{O}_{\text {water }}$ and $\mathrm{N}-\mathrm{H} \cdots$ Osulfato hydrogen bonds. Together, a series of $\mathrm{N}-\mathrm{H} \cdots \mathrm{O}$ and $\mathrm{O}-\mathrm{H} \cdots \mathrm{O}$ hydrogen bonds, involving the $\mathrm{O}$ atoms of the thiosulfate ions and the water molecules as acceptors and the hydrogen atoms of the piperazinium ions and the water molecules as donors, form a three-dimensional supramolecuar structure. Within this framework there are a number of intra- and intermolecular $\mathrm{C}-\mathrm{H} \cdots \mathrm{O}$ and $\mathrm{C}-\mathrm{H} \cdots \mathrm{S}$ contacts present.

\section{Chemical context}

Over the last few decades, a large number of amine-templated metal complexes and compounds with extended structures have been synthesized in the presence of a number of inorganic anions (Férey, 2008). One series of anions, namely the sulfur-containing oxoanions, and in particular sulfates and sulfites, are widely used in the synthesis of higher dimensional inorganic compounds because of their multidentate coordination capacity towards metal ions (Rao et al., 2006). In these examples, the anions bind to the metal cations through the oxygen atoms. The thiosulfate ion is a new example of an sulfur oxoanion used in amine-templated synthesis, although the reactivity of this ligand is less than that of the sulfate and sulfite ions. In this heteroatomic ligand, the terminal S atom, as well as the $\mathrm{O}$ atoms, can bind to a range of metal ions. However, the long $\mathrm{S}-\mathrm{S}$ bond is unstable under acidic conditions or at high temperature. Hence, the thiosulfate anion has not, to date, been explored extensively as a network-building unit for higher dimensional structures (Paul et al., 2011). Despite these stability complications, Baggio and co-workers have synthesized a few molecular and one-dimensional structures containing thiosulfate anions that are connected to the metal through oxygen as well as sulfur atoms (Baggio et al., 1996, 1997; Freire et al., 2001; Harvey et al., 2004). Our continuing synthetic efforts using the thiosulfate anion have resulted in the synthesis of some new three-dimensional structures in the family of cadmium-thiosulfate hybrid compounds formed in the presence of organic linkers (Paul et $a l ., 2009 a, b, 2010)$. It is noteworthy that all of the reported metal-thiosulfate compounds are synthesized in the presence of nitrogen-containing aromatic organic linkers. Aromatic 
ligands play a dual role in metal-thiosulfate formation as they increase the dimensionality of the local structure and increase structure stabilization via secondary interactions, such as hydrogen bonds. Recently, Natarajan and co-workers (Karthik \& Natarajan, 2016) have reported on some three-dimensional zinc-thiosulfate hybrid structures with aromatic N-donor organic linkers. Metal-thiosulfate compounds prepared in the presence of aliphatic amines are, however, rare (Paul, 2016) and require investigation. The title compound, is the first example of an aliphatic-amine-templated zinc thiosulfate compound. Its synthesis and crystal structure are reported on herein.

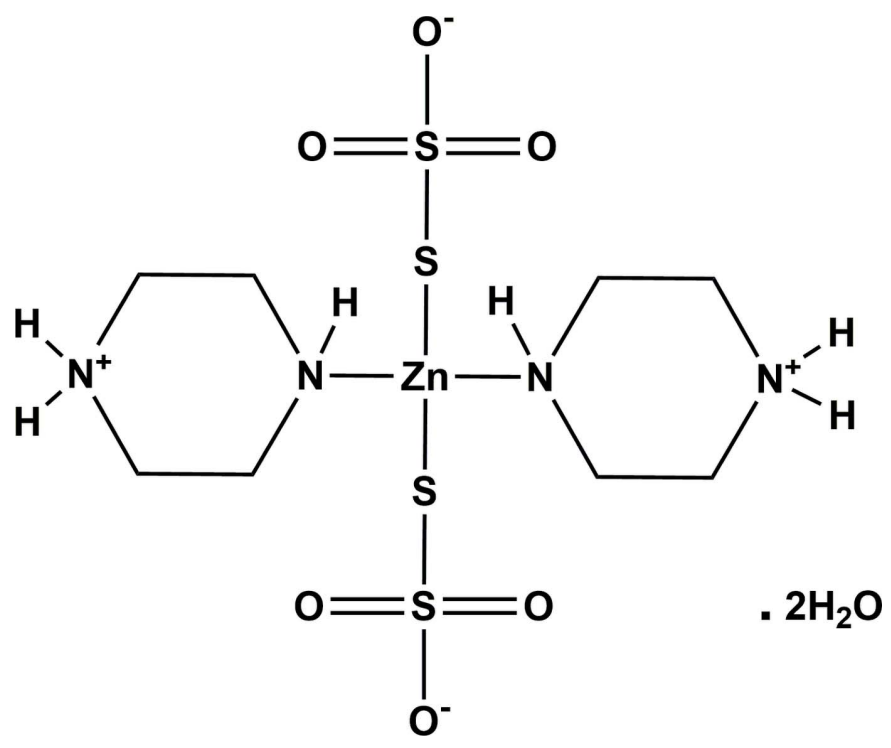

\section{Structural commentary}

The molecular structure of the title compound is illustrated in Fig. 1. In the complex, the $\mathrm{Zn}^{2+}$ ion is coordinated by two sulfur atoms of the thiosulfate ligands (S1 and S3) and two

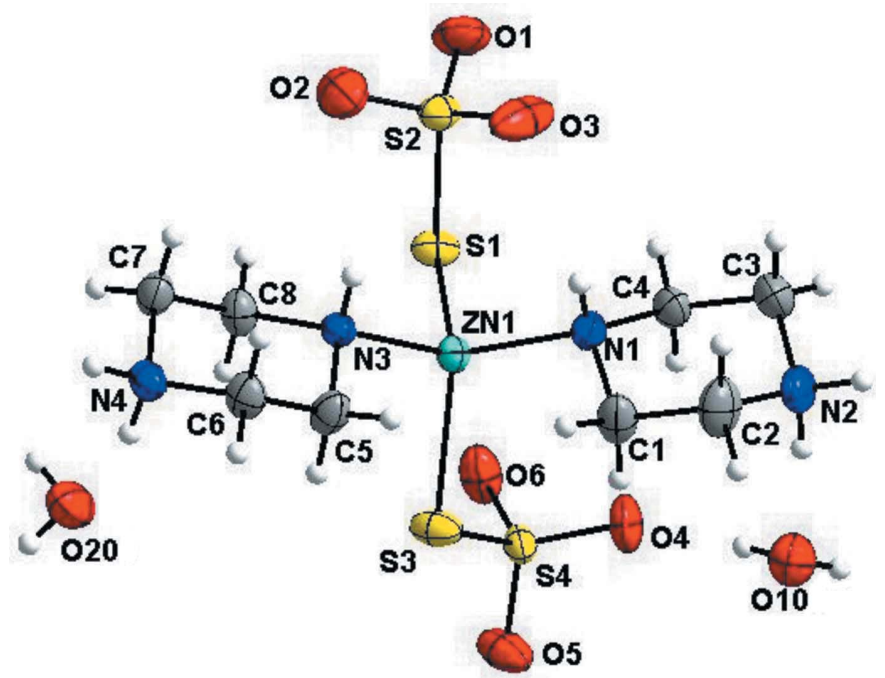

Figure 1

The asymmetric unit of the title compound, with atom labelling and showing $50 \%$ probability displacement ellipsoids.
Table 1

Hydrogen-bond geometry $\left(\AA,^{\circ}\right)$.

\begin{tabular}{|c|c|c|c|c|}
\hline$D-\mathrm{H} \cdots A$ & $D-\mathrm{H}$ & $\mathrm{H} \cdots A$ & $D \cdots A$ & $D-\mathrm{H} \cdots A$ \\
\hline $\mathrm{N} 1-\mathrm{H} 1 \cdots \mathrm{O} 2^{\mathrm{i}}$ & $0.83(2)$ & $2.25(2)$ & $3.041(2)$ & $160(2)$ \\
\hline $\mathrm{N} 2-\mathrm{H} 2 A N \cdots \mathrm{O} 6^{\mathrm{ii}}$ & $0.95(3)$ & $1.80(3)$ & $2.730(2)$ & $166(2)$ \\
\hline $\mathrm{N} 2-\mathrm{H} 2 B N \cdots \mathrm{O} 10$ & $0.93(2)$ & $1.89(2)$ & $2.811(3)$ & $170(2)$ \\
\hline $\mathrm{N} 3-\mathrm{H} 3 \cdots \mathrm{O}^{\mathrm{i}}$ & $0.84(2)$ & $2.03(2)$ & $2.853(2)$ & $166(2)$ \\
\hline $\mathrm{N} 4-\mathrm{H} 4 A N \cdots \mathrm{O} 20$ & $0.87(2)$ & $2.09(2)$ & $2.892(2)$ & $154(2)$ \\
\hline $\mathrm{N} 4-\mathrm{H} 4 B N \cdots \mathrm{O} 5^{\mathrm{iii}}$ & $0.94(2)$ & $1.84(2)$ & $2.763(2)$ & $169(2)$ \\
\hline $\mathrm{O} 10-\mathrm{H} 10 A \cdots \mathrm{O} 4$ & $0.85(3)$ & $1.94(4)$ & $2.780(2)$ & $175(3)$ \\
\hline $\mathrm{O} 10-\mathrm{H} 10 B \cdots \mathrm{O} 1^{\mathrm{iv}}$ & $0.76(3)$ & $2.02(3)$ & $2.777(2)$ & $176(4)$ \\
\hline $\mathrm{O} 20-\mathrm{H} 20 A \cdots \mathrm{O} 1^{\mathrm{v}}$ & $0.82(3)$ & $2.02(3)$ & $2.804(2)$ & $163(3)$ \\
\hline $\mathrm{O} 20-\mathrm{H} 20 B \cdots \mathrm{O} 5^{\mathrm{vi}}$ & $0.74(3)$ & $2.17(3)$ & $2.866(2)$ & $158(3)$ \\
\hline $\mathrm{C} 3-\mathrm{H} 3 A \cdots \mathrm{O}^{\mathrm{iv}}$ & 0.97 & 2.45 & $3.221(2)$ & 136 \\
\hline $\mathrm{C} 4-\mathrm{H} 4 A \cdots \mathrm{O} 3$ & 0.97 & 2.49 & $3.175(3)$ & 128 \\
\hline $\mathrm{C} 4-\mathrm{H} 4 B \cdots \mathrm{O} 4$ & 0.97 & 2.54 & $3.463(2)$ & 159 \\
\hline $\mathrm{C} 5-\mathrm{H} 5 B \cdots \mathrm{S} 3$ & 0.97 & 2.86 & $3.453(2)$ & 120 \\
\hline $\mathrm{C} 8-\mathrm{H} 8 B \cdots \mathrm{O} 2$ & 0.97 & 2.50 & $3.318(2)$ & 142 \\
\hline
\end{tabular}

Symmetry codes: (i) $-x+1,-y+1,-z$; (ii) $x+1, y, z$; (iii) $x, y+1, z$; (iv) $-x+1,-y,-z ;$ (v) $-x,-y+1,-z ;($ vi) $-x,-y+1,-z+1$.

nitrogen atoms from the piperazinium ions ( $\mathrm{N} 1$ and N3), in an approximately tetrahedral geometry $\left(\mathrm{ZnS}_{2} \mathrm{~N}_{2}, \mathrm{CN}=4\right)$. The $\mathrm{Zn}-\mathrm{S}$ bond lengths are 2.2927 (4) $\AA$ for $\mathrm{Zn} 1-\mathrm{S} 1$ and 2.3324 (4) $\AA$ for $\mathrm{Zn} 1-\mathrm{S} 3$. The $\mathrm{Zn}-\mathrm{N}$ bond lengths are 2.0879 (13) $\AA$ for $\mathrm{Zn} 1-\mathrm{N} 1$ and 2.0727 (12) $\AA$ for $\mathrm{Zn} 1-\mathrm{N} 3$. The $\mathrm{N} / \mathrm{S}-\mathrm{Zn} 1-\mathrm{S} / \mathrm{N}$ bond angles lie in the range 101.24 (4) to $116.79(2)^{\circ}$, confirming the tetrahedral nature of the zinc ions. Within the two thiosulfate ligands, the $\mathrm{S}-\mathrm{S}$ bond lengths are 2.0511 (5) $\AA$ for S1 - S2 and 2.0332 (5) $\AA$ for S3-S4. The SO bond lengths vary from 1.4437 (14) to 1.4623 (13) $\AA$, while the $\mathrm{O}-\mathrm{S}-\mathrm{O}$ angles vary from $104.53(5)$ to $112.85(10)^{\circ}$, which is indicative of a fairly regular tetrahedral arrangement. In the molecular unit, the two thiosulfate units are bonded to the zinc(II) ion only through the terminal S atoms, and the oxygen atoms are uncoordinated. In addition, only one nitrogen atom of each piperazinium ion is bonded to the zinc(II) ion, the second being diprotonated in each case.

\section{Supramolecular features}

The supramolecular architecture (Fig. 2) arises from a threedimensional network of $\mathrm{N}-\mathrm{H} \cdots \mathrm{O}$ and $\mathrm{O}-\mathrm{H} \cdots \mathrm{O}$ hydrogen bonds involving the uncoordinated oxygen atoms of the thiosulfate ligands, the protonated piperazine units and the lattice water molecules (Table 1). These intermolecular interactions lead to the formation of a supramolecular framework. Within this framework there are a number of intra- and intermolecular $\mathrm{C}-\mathrm{H} \cdots \mathrm{O}$ and $\mathrm{C}-\mathrm{H} \cdots \mathrm{S}$ contacts present (Table 1).

\section{Database survey}

A search of the Cambridge Structural Database (CSD, Version 35.9, last update May 2017; Groom et al., 2016) for zinc-thiosulfato complexes gave 12 hits, all involving aromatic amines and/or thioureas. Díaz de Vivar et al. (2006) have described a molecular zinc-thiosulfate complex prepared in the presence of a tridentate aromatic ligand, viz. aqua(thio- 


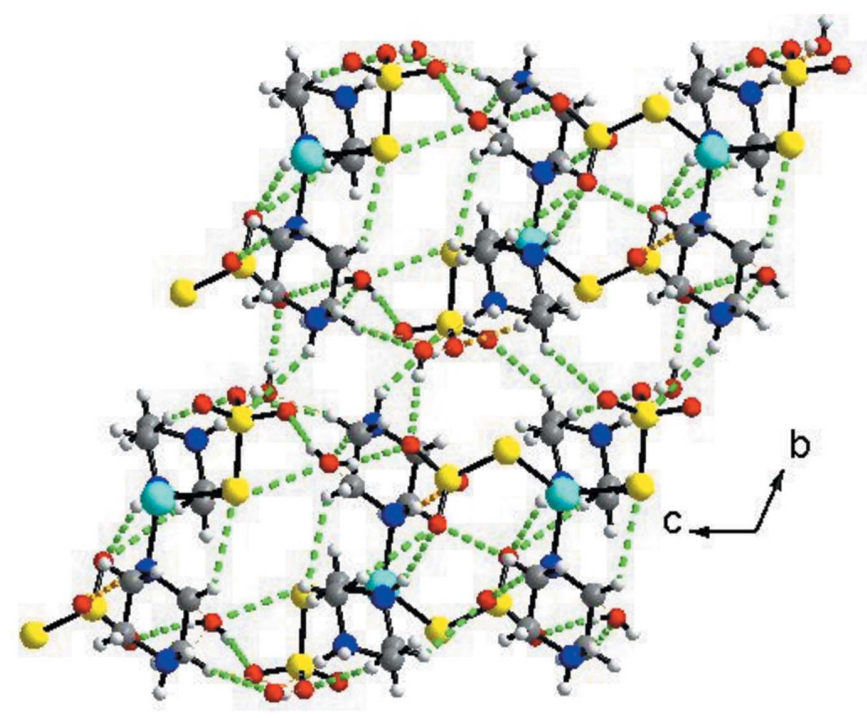

Figure 2

A view along the $a$ axis of the crystal packing of the title compound. The hydrogen bonds are shown as dashed lines (see Table 1).

sulfato- $\kappa O, S)\left[2,4,6\right.$-tris(2-pyridyl)-1,3,5-triazine- $\left.N, N^{\prime}, N^{\prime \prime}\right]$ zinc(II) hemihydrate (CSD refcode: WEHTOT). The thiosulfate ligand is coordinated to the zinc ions through $\mathrm{S}$ and $\mathrm{O}$ atoms, forming octahedral zinc centres. In addition, a zincthiosulfate complex containing both one-dimensional cationic and anionic chains has been reported by the same authors, viz. catena-[( $\mu^{2}-4,4^{\prime}$-bipyridine- $\left.\kappa N, N^{\prime}\right)$ tetraaquazinc(II) $\operatorname{bis}\left(\mu^{2}\right.$ $4,4^{\prime}$-bipyridine- $\left.\kappa N, N^{\prime}\right)\left(\mu^{2}\right.$-thiosulfato- $\left.\kappa O, S\right)$ bis(thiosulfato$\kappa S$ )dizinc(II) dihydrate] [PEYLEL; Díaz de Vivar et al., 2007). Both types of chain contain 4,4'-bipyridine ligands as linkers.

Karthik \& Natarajan (2016) have recently reported four higher-dimensional zinc-thiosulfate compounds synthesized in the presence of various aromatic ligands, viz. catena-[bis $(\mu$ 4,4'-bipyridine)bis( $\mu$-thiosulfato)dizinc] (IJUWER), catena[( $\mu$-4,4'-propane-1,3-diyldipyridine)( $\mu$-thiosulfato)zinc] (IJUWIV), and catena-[bis ( $\mu$-4,4'-ethene-1,2-diyldipyridine) bis $(\mu$ thiosulfato)dizinc dihydrate] (IJUWOB) and catena[bis $(\mu$-4,4'-ethane-1,2-diyldipyridine)bis( $\mu$-thiosulfato)dizinc ( $\mu$-4,4'-ethane-1,2-diyldipyridine)( $\mu$-thiosulfato)zinc trihydrate] (IJUWUH).

A number of molecular cadmium-thiosulfate and manganese-thiosulfate structures have been reported by Baggio and co-workers (Baggio et al., 1996, 1997; Freire et al., 2001; Harvey et al., 2004). They were synthesized in the presence of 2,2'-bipyridine or 1,10-phenanthroline.

There are a few examples in which zero-dimensional cadmium-thiosulfate compounds form simple dinuclear complexes, in which the thiosulfate unit is bound to the metal through both the sulfur and the oxygen atoms. As expected, the structures are stabilized through $\mathrm{C}-\mathrm{H} \cdots \mathrm{O}$ hydrogenbonding interactions and $\pi-\pi$ interactions. One cadmium thiosulfate compound, bis(propane-1,3-diamine)(thiosulfato)cadmium (CSD refcode: ORUJOC), which was reported recently, was isolated in the presence of the aliphatic amine 1,3-diaminopropane (Paul, 2016). One molecular piper-

Table 2

Experimental details.

Crystal data

Chemical formula

$M_{\mathrm{r}}$

Crystal system, space group

Temperature (K)

$a, b, c(\AA)$

$\alpha, \beta, \gamma\left({ }^{\circ}\right)$

$V\left(\AA^{3}\right)$

$Z$

Radiation type

$\mu\left(\mathrm{mm}^{-1}\right)$

Crystal size (mm)

Data collection

Diffractometer

Absorption correction

$T_{\min }, T_{\max }$

No. of measured, independent and observed $[I>2 \sigma(I)]$ reflections

$R_{\text {int }}$

$(\sin \theta / \lambda)_{\max }\left(\AA^{-1}\right)$

Refinement

$R\left[F^{2}>2 \sigma\left(F^{2}\right)\right], w R\left(F^{2}\right), S$

No. of reflections

No. of parameters

$\mathrm{H}$-atom treatment

$\Delta \rho_{\max }, \Delta \rho_{\min }\left(\mathrm{e} \AA^{-3}\right)$

Computer programs: SMART and SAINT (Bruker, 2000), SHELXS97 (Sheldrick, 2008), SHELXL2016/6 (Sheldrick, 2015), ORTEP-3 for Windows (Farrugia, 2012), PLATON (Spek, 2009) and publCIF (Westrip, 2010).

azinium thiosulfate monohydrate structure has been reported, (piperazinediium thiosulfate monohydrate; CSD refcode: AROWUA; Srinivasan et al., 2011), in which the protonated aliphatic amine and thiosulfate units are linked together through extensive hydrogen bonds. It is noteworthy that there are no previous examples in the literature of zinc-thiosulfate structures that crystallize in the presence of aliphatic amines.

\section{Synthesis and crystallization}

$\mathrm{Zn}\left(\mathrm{NO}_{3}\right)_{2} \cdot 6 \mathrm{H}_{2} \mathrm{O}(0.297 \mathrm{~g}, 1 \mathrm{mmol})$ was dissolved in $5 \mathrm{ml}$ distilled water. Then $\left(\mathrm{NH}_{4}\right)_{2} \mathrm{~S}_{2} \mathrm{O}_{3}(0.296 \mathrm{~g}, 2 \mathrm{mmol})$ was added to the solution, which was stirred for $15 \mathrm{~min}$. Piperazine $(0.172 \mathrm{~g}, 2 \mathrm{mmol})$ was dissolved separately in distilled water $(5 \mathrm{ml})$ and the solution poured into the initial reaction mixture until the $\mathrm{pH}$ was 8 . The resulting solution was left undisturbed and after 1 week, colourless block-shaped crystals were obtained. The product was filtered and washed with cold water. The yield was approximately $85 \%$ based on $\mathrm{Zn}$ metal. Elemental analysis calculated for $\mathrm{C}_{8} \mathrm{H}_{26} \mathrm{~N}_{4} \mathrm{O}_{8} \mathrm{~S}_{4} \mathrm{Zn}$ : C 19.20, H 5.24, N 11.20\%; found: C 19.27, H 5.29, N 11.16\%.

\section{Refinement}

Crystal data, data collection and structure refinement details are summarized in Table 2. The $\mathrm{NH}, \mathrm{NH}_{2}$ and water $\mathrm{H}$ atoms 
were located in difference-Fourier maps and freely refined. The $\mathrm{C}$-bound $\mathrm{H}$ atoms were included in calculated positions and refined as riding: $\mathrm{C}-\mathrm{H}=0.97 \AA$ with $U_{\text {iso }}(\mathrm{H})=1.2 U_{\text {eq }}(\mathrm{C})$.

\section{Acknowledgements}

The author thanks Professor S. Natarajan for providing facilities.

\section{Funding information}

The author thanks the SERB and DST, India, for research grants.

\section{References}

Baggio, R., Baggio, S., Pardo, M. I. \& Garland, M. T. (1996). Acta Cryst. C52, 1939-1942.

Baggio, S., Pardo, M. I., Baggio, R. \& Garland, M. T. (1997). Acta Cryst. C53, 727-729.

Bruker (2000). SMART, SAINT and SADABS. Bruker AXS Inc., Madison, Wisconsin, USA.

Díaz de Vivar, M. E., Baggio, S. \& Baggio, R. (2006). Acta Cryst. C62, m192-m194.

Díaz de Vivar, M. E., Baggio, S., Garland, M. T. \& Baggio, R. (2007). Acta Cryst. C63, m123-m125.
Farrugia, L. J. (2012). J. Appl. Cryst. 45, 849-854.

Férey, G. (2008). Chem. Soc. Rev. 37, 191-214.

Freire, E., Baggio, S., Baggio, R. \& Mombrú, A. (2001). Acta Cryst. C57, 14-17.

Groom, C. R., Bruno, I. J., Lightfoot, M. P. \& Ward, S. C. (2016). Acta Cryst. B72, 171-179.

Harvey, M., Baggio, S., Pardo, H. \& Baggio, R. (2004). Acta Cryst. C60, m79-m81.

Karthik, R. \& Natarajan, S. (2016). Cryst. Growth Des. 16, 2239-2248.

Paul, A. K. (2016). J. Mol. Struct. 1125, 696-704.

Paul, A. K., Karthik, R. \& Natarajan, S. (2011). Cryst. Growth Des. 11, 5741-5749.

Paul, A. K., Madras, G. \& Natarajan, S. (2009a). CrystEngComm, 11, 55-57.

Paul, A. K., Madras, G. \& Natarajan, S. (2009b). Phys. Chem. Chem. Phys. 11, 11285-11296.

Paul, A. K., Madras, G. \& Natarajan, S. (2010). Dalton Trans. 39, 2263-2279.

Rao, C. N. R., Behera, J. N. \& Dan, M. (2006). Chem. Soc. Rev. 35, 375-387.

Sheldrick, G. M. (2008). Acta Cryst. A64, 112-122.

Sheldrick, G. M. (2015). Acta Cryst. C71, 3-8.

Spek, A. L. (2009). Acta Cryst. D65, 148-155.

Srinivasan, B. R., Naik, A. R., Dhuri, S. N., Näther, C. \& Bensch, W. (2011). J. Chem. Sci. 123, 55-61.

Westrip, S. P. (2010). J. Appl. Cryst. 43, 920-925. 


\section{supporting information}

Acta Cryst. (2018). E74, 176-179 [https://doi.org/10.1107/S2056989018000555]

\section{Crystal structure of bis(piperazin-1-ium- $\kappa N^{4}$ ) bis(thiosulfato- $\left.k S\right)$ zinc(II)} dihydrate

\section{Avijit Kumar Paul}

\section{Computing details}

Data collection: SMART (Bruker, 2000); cell refinement: SAINT (Bruker, 2000); data reduction: SAINT (Bruker, 2000); program(s) used to solve structure: SHELXS97 (Sheldrick, 2008); program(s) used to refine structure: SHELXL2016/6 (Sheldrick, 2015); molecular graphics: ORTEP-3 for Windows (Farrugia, 2012); software used to prepare material for publication: SHELXL2016/6 (Sheldrick, 2015), PLATON (Spek, 2009) and publCIF (Westrip, 2010).

Bis(piperazin-1-ium- $\kappa N^{4}$ )bis(thiosulfato- $\kappa S$ )zinc(II) dihydrate

\section{Crystal data}

$\left[\mathrm{Zn}\left(\mathrm{C}_{4} \mathrm{H}_{11} \mathrm{~N}_{2}\right)_{2}\left(\mathrm{~S}_{2} \mathrm{O}_{3}\right)_{2}\right] \cdot 2 \mathrm{H}_{2} \mathrm{O}$

$M_{r}=499.94$

Triclinic, $P \overline{1}$

$a=8.7631(1) \AA$

$b=10.5623(2) \AA$

$c=11.6072(2) \AA$

$\alpha=113.736(1)^{\circ}$

$\beta=98.761(1)^{\circ}$

$\gamma=91.472(1)^{\circ}$

$V=967.49(3) \AA^{3}$

\section{Data collection}

Bruker SMART APEX CCD area detector diffractometer

Radiation source: fine-focus sealed tube Graphite monochromator $\varphi$ and $\omega$ scans

Absorption correction: multi-scan

(SADABS; Bruker, 2000)

$T_{\min }=0.700, T_{\max }=0.768$

Refinement

Refinement on $F^{2}$

Least-squares matrix: full

$R\left[F^{2}>2 \sigma\left(F^{2}\right)\right]=0.032$

$w R\left(F^{2}\right)=0.079$

$S=1.01$

7607 reflections

266 parameters

0 restraints
$Z=2$

$F(000)=520$

$D_{\mathrm{x}}=1.716 \mathrm{Mg} \mathrm{m}^{-3}$

Mo $K \alpha$ radiation, $\lambda=0.71073 \AA$

Cell parameters from 3790 reflections

$\theta=2.0-26.0^{\circ}$

$\mu=1.74 \mathrm{~mm}^{-1}$

$T=293 \mathrm{~K}$

Block, colorless

$0.22 \times 0.18 \times 0.16 \mathrm{~mm}$

19938 measured reflections

7607 independent reflections

5927 reflections with $I>2 \sigma(I)$

$R_{\text {int }}=0.027$

$\theta_{\max }=33.7^{\circ}, \theta_{\min }=2.0^{\circ}$

$h=-13 \rightarrow 13$

$k=-16 \rightarrow 9$

$l=-18 \rightarrow 17$

Primary atom site location: structure-invariant direct methods

Secondary atom site location: difference Fourier map

Hydrogen site location: mixed

$\mathrm{H}$ atoms treated by a mixture of independent and constrained refinement 
$w=1 /\left[\sigma^{2}\left(F_{\mathrm{o}}^{2}\right)+(0.040 P)^{2}\right]$

where $P=\left(F_{\mathrm{o}}^{2}+2 F_{\mathrm{c}}{ }^{2}\right) / 3$

$(\Delta / \sigma)_{\max }<0.001$

$$
\Delta \rho_{\max }=0.47 \mathrm{e} \AA^{-3}
$$

\section{Special details}

Geometry. All esds (except the esd in the dihedral angle between two 1.s. planes) are estimated using the full covariance matrix. The cell esds are taken into account individually in the estimation of esds in distances, angles and torsion angles; correlations between esds in cell parameters are only used when they are defined by crystal symmetry. An approximate (isotropic) treatment of cell esds is used for estimating esds involving l.s. planes.

Fractional atomic coordinates and isotropic or equivalent isotropic displacement parameters $\left(\hat{A}^{2}\right)$

\begin{tabular}{|c|c|c|c|c|}
\hline & $x$ & $y$ & $z$ & $U_{\text {iso }} * / U_{\text {eq }}$ \\
\hline Zn1 & $0.36403(2)$ & $0.36678(2)$ & $0.17364(2)$ & $0.02307(5)$ \\
\hline S1 & $0.19769(4)$ & $0.23705(4)$ & $-0.01347(4)$ & $0.02921(8)$ \\
\hline S2 & $0.28149(4)$ & $0.31755(4)$ & $-0.12806(4)$ & $0.02866(8)$ \\
\hline $\mathrm{S} 3$ & $0.31486(6)$ & $0.34577(4)$ & $0.35739(4)$ & $0.03543(10)$ \\
\hline S4 & $0.27051(4)$ & $0.13632(4)$ & $0.29154(4)$ & $0.02567(8)$ \\
\hline $\mathrm{O} 1$ & $0.22237(19)$ & $0.21735(14)$ & $-0.25891(12)$ & $0.0508(4)$ \\
\hline $\mathrm{O} 2$ & $0.22392(19)$ & 0.45077 (14) & $-0.10572(15)$ & $0.0541(4)$ \\
\hline $\mathrm{O} 3$ & $0.44978(15)$ & 0.32601 (19) & $-0.09909(15)$ & $0.0612(4)$ \\
\hline $\mathrm{O} 4$ & $0.40860(15)$ & $0.06807(14)$ & $0.25701(14)$ & $0.0486(3)$ \\
\hline O5 & $0.2234(2)$ & $0.11500(13)$ & $0.39847(14)$ & $0.0544(4)$ \\
\hline O6 & $0.14635(14)$ & $0.09045(13)$ & $0.18082(13)$ & $0.0435(3)$ \\
\hline N1 & $0.59630(14)$ & $0.33597(13)$ & $0.16071(13)$ & $0.0261(2)$ \\
\hline H1 & $0.627(2)$ & $0.3885(19)$ & $0.1297(18)$ & $0.033(5)^{*}$ \\
\hline $\mathrm{N} 2$ & $0.86568(17)$ & $0.19550(17)$ & $0.20334(16)$ & $0.0381(3)$ \\
\hline $\mathrm{H} 2 \mathrm{AN}$ & $0.968(3)$ & $0.172(2)$ & $0.194(2)$ & $0.053(6)^{*}$ \\
\hline $\mathrm{H} 2 \mathrm{BN}$ & $0.831(3)$ & $0.149(2)$ & $0.249(2)$ & $0.058(7)^{*}$ \\
\hline N3 & $0.34859(14)$ & $0.57724(12)$ & $0.22385(12)$ & $0.0225(2)$ \\
\hline $\mathrm{H} 3$ & $0.396(2)$ & $0.5979(17)$ & $0.1754(17)$ & $0.024(4)^{*}$ \\
\hline N4 & $0.24360(17)$ & $0.84404(13)$ & $0.37115(13)$ & $0.0298(3)$ \\
\hline H4AN & $0.186(2)$ & $0.8219(19)$ & $0.4161(19)$ & $0.037(5)^{*}$ \\
\hline $\mathrm{H} 4 \mathrm{BN}$ & $0.229(2)$ & $0.937(2)$ & $0.3879(19)$ & $0.043(5)^{*}$ \\
\hline $\mathrm{C} 1$ & $0.69716(19)$ & $0.37986(18)$ & $0.28807(16)$ & $0.0381(4)$ \\
\hline $\mathrm{H} 1 \mathrm{~A}$ & 0.654430 & 0.334114 & 0.335305 & $0.046^{*}$ \\
\hline H1B & 0.696156 & 0.479233 & 0.335289 & $0.046^{*}$ \\
\hline $\mathrm{C} 2$ & $0.8636(2)$ & $0.34663(19)$ & $0.2805(2)$ & $0.0454(5)$ \\
\hline $\mathrm{H} 2 \mathrm{~A}$ & 0.911324 & 0.399270 & 0.241316 & $0.055^{*}$ \\
\hline $\mathrm{H} 2 \mathrm{~B}$ & 0.922320 & 0.372326 & 0.365901 & $0.055^{*}$ \\
\hline $\mathrm{C} 3$ & $0.7752(2)$ & $0.1542(2)$ & $0.07222(18)$ & $0.0421(4)$ \\
\hline $\mathrm{H} 3 \mathrm{~A}$ & 0.777441 & 0.055408 & 0.022780 & $0.051^{*}$ \\
\hline H3B & 0.821264 & 0.203770 & 0.029594 & $0.051^{*}$ \\
\hline $\mathrm{C} 4$ & $0.60942(19)$ & $0.18742(16)$ & $0.07979(15)$ & $0.0315(3)$ \\
\hline $\mathrm{H} 4 \mathrm{~A}$ & 0.552976 & 0.164170 & -0.005869 & $0.038^{*}$ \\
\hline $\mathrm{H} 4 \mathrm{~B}$ & 0.561198 & 0.129930 & 0.114233 & $0.038^{*}$ \\
\hline $\mathrm{C} 5$ & $0.4203(2)$ & $0.66344(15)$ & $0.35818(15)$ & $0.0327(3)$ \\
\hline H5A & 0.528902 & 0.647499 & 0.370824 & $0.039 *$ \\
\hline $\mathrm{H} 5 \mathrm{~B}$ & 0.370312 & 0.634514 & 0.413833 & $0.039 *$ \\
\hline
\end{tabular}




$\begin{array}{lllll}\text { C6 } & 0.40821(19) & 0.81761(15) & 0.39612(16) & 0.0330(3) \\ \text { H6A } & 0.450934 & 0.868622 & 0.486215 & 0.040^{*} \\ \text { H6B } & 0.467790 & 0.849836 & 0.347563 & 0.040^{*} \\ \text { C7 } & 0.17408(19) & 0.76190(16) & 0.23399(16) & 0.0333(3) \\ \text { H7A } & 0.227849 & 0.791358 & 0.180529 & 0.040^{*} \\ \text { H7B } & 0.065948 & 0.778454 & 0.219288 & 0.040^{*} \\ \text { C8 } & 0.18624(17) & 0.60873(15) & 0.19857(16) & 0.0308(3) \\ \text { H8A } & 0.124751 & 0.578258 & 0.247102 & 0.037^{*} \\ \text { H8B } & 0.143694 & 0.557112 & 0.108512 & 0.037^{*} \\ \text { O10 } & 0.72416(18) & 0.05969(17) & 0.32966(15) & 0.0460(3) \\ \text { H10A } & 0.629(4) & 0.060(3) & 0.303(3) & 0.098(11)^{*} \\ \text { H10B } & 0.742(3) & -0.015(3) & 0.313(3) & 0.074(10)^{*} \\ \text { O20 } & -0.01440(19) & 0.7318(2) & 0.44092(18) & 0.0557(4) \\ \text { H20A } & -0.082(4) & 0.729(3) & 0.383(3) & 0.092(11)^{*} \\ \text { H20B } & -0.048(3) & 0.776(3) & 0.497(3) & 0.070(9)^{*} \\ \end{array}$

Atomic displacement parameters $\left(\AA^{2}\right)$

\begin{tabular}{|c|c|c|c|c|c|c|}
\hline & $U^{11}$ & $U^{22}$ & $U^{33}$ & $U^{12}$ & $U^{13}$ & $U^{23}$ \\
\hline Zn1 & $0.02461(8)$ & $0.02117(8)$ & $0.02345(8)$ & $0.00381(6)$ & $0.00452(6)$ & $0.00900(6)$ \\
\hline S1 & $0.02902(18)$ & $0.03198(19)$ & $0.02573(17)$ & $-0.00689(14)$ & $0.00212(13)$ & $0.01250(15)$ \\
\hline $\mathrm{S} 2$ & $0.02756(17)$ & $0.0354(2)$ & $0.02670(18)$ & $0.00130(14)$ & 0.00454 (13) & $0.01668(15)$ \\
\hline S3 & $0.0560(3)$ & $0.02537(18)$ & $0.02544(18)$ & $-0.00262(17)$ & 0.01059 (17) & $0.01015(15)$ \\
\hline S4 & $0.02671(16)$ & $0.02414(17)$ & $0.03090(18)$ & $0.00813(13)$ & $0.01197(13)$ & $0.01342(14)$ \\
\hline $\mathrm{O} 1$ & $0.0746(10)$ & $0.0502(8)$ & $0.0237(6)$ & $0.0060(7)$ & $0.0030(6)$ & $0.0132(5)$ \\
\hline $\mathrm{O} 2$ & $0.0744(10)$ & $0.0403(7)$ & $0.0630(9)$ & $0.0151(7)$ & $0.0259(8)$ & $0.0316(7)$ \\
\hline $\mathrm{O} 3$ & $0.0291(6)$ & $0.1148(13)$ & $0.0692(10)$ & $-0.0021(7)$ & $0.0074(6)$ & $0.0689(10)$ \\
\hline $\mathrm{O} 4$ & $0.0356(7)$ & $0.0524(8)$ & $0.0618(9)$ & $0.0251(6)$ & $0.0179(6)$ & $0.0229(7)$ \\
\hline O5 & $0.0985(12)$ & $0.0319(6)$ & $0.0513(8)$ & $0.0170(7)$ & $0.0449(8)$ & $0.0242(6)$ \\
\hline O6 & $0.0308(6)$ & $0.0364(7)$ & $0.0466(7)$ & $0.0042(5)$ & $0.0010(5)$ & $0.0021(5)$ \\
\hline N1 & $0.0255(6)$ & $0.0260(6)$ & $0.0290(6)$ & $0.0060(5)$ & $0.0052(5)$ & $0.0132(5)$ \\
\hline $\mathrm{N} 2$ & $0.0270(7)$ & $0.0479(9)$ & $0.0476(9)$ & $0.0152(6)$ & $0.0120(6)$ & $0.0253(7)$ \\
\hline N3 & $0.0239(5)$ & $0.0217(5)$ & $0.0234(6)$ & $0.0036(4)$ & $0.0068(4)$ & $0.0097(5)$ \\
\hline N4 & $0.0379(7)$ & $0.0219(6)$ & $0.0331(7)$ & $0.0078(5)$ & $0.0141(6)$ & $0.0119(5)$ \\
\hline $\mathrm{C} 1$ & $0.0325(8)$ & $0.0364(9)$ & $0.0334(8)$ & $0.0085(7)$ & $-0.0011(6)$ & $0.0040(7)$ \\
\hline $\mathrm{C} 2$ & $0.0257(8)$ & $0.0450(10)$ & $0.0570(12)$ & $0.0036(7)$ & $-0.0024(8)$ & $0.0156(9)$ \\
\hline $\mathrm{C} 3$ & $0.0425(9)$ & $0.0500(11)$ & $0.0373(9)$ & $0.0219(8)$ & $0.0164(7)$ & $0.0172(8)$ \\
\hline $\mathrm{C} 4$ & $0.0335(8)$ & $0.0311(8)$ & $0.0273(7)$ & $0.0101(6)$ & $0.0061(6)$ & $0.0087(6)$ \\
\hline $\mathrm{C} 5$ & $0.0377(8)$ & $0.0255(7)$ & $0.0294(7)$ & $0.0066(6)$ & $-0.0024(6)$ & $0.0085(6)$ \\
\hline C6 & $0.0351(8)$ & $0.0231(7)$ & $0.0342(8)$ & $0.0025(6)$ & $0.0018(6)$ & $0.0065(6)$ \\
\hline $\mathrm{C} 7$ & $0.0349(8)$ & $0.0307(8)$ & $0.0336(8)$ & $0.0105(6)$ & $0.0027(6)$ & $0.0133(6)$ \\
\hline $\mathrm{C} 8$ & $0.0248(7)$ & $0.0269(7)$ & $0.0361(8)$ & $0.0044(5)$ & $0.0028(6)$ & $0.0091(6)$ \\
\hline $\mathrm{O} 10$ & $0.0428(8)$ & $0.0457(8)$ & $0.0492(8)$ & $0.0068(6)$ & $0.0067(6)$ & $0.0197(7)$ \\
\hline $\mathrm{O} 20$ & $0.0445(8)$ & $0.0857(12)$ & $0.0441(9)$ & $0.0215(8)$ & $0.0168(7)$ & $0.0300(9)$ \\
\hline
\end{tabular}


Geometric parameters $\left(\AA,{ }^{\circ}\right)$

\begin{tabular}{|c|c|c|c|}
\hline $\mathrm{Zn} 1-\mathrm{N} 3$ & $2.0727(12)$ & $\mathrm{N} 4-\mathrm{H} 4 \mathrm{BN}$ & $0.93(2)$ \\
\hline $\mathrm{Zn} 1-\mathrm{N} 1$ & $2.0879(13)$ & $\mathrm{C} 1-\mathrm{C} 2$ & $1.516(2)$ \\
\hline $\mathrm{Zn} 1-\mathrm{S} 1$ & $2.2927(4)$ & $\mathrm{C} 1-\mathrm{H} 1 \mathrm{~A}$ & 0.9700 \\
\hline $\mathrm{Zn} 1-\mathrm{S} 3$ & $2.3324(4)$ & $\mathrm{C} 1-\mathrm{H} 1 \mathrm{~B}$ & 0.9700 \\
\hline $\mathrm{S} 1-\mathrm{S} 2$ & $2.0511(5)$ & $\mathrm{C} 2-\mathrm{H} 2 \mathrm{~A}$ & 0.9700 \\
\hline $\mathrm{S} 2-\mathrm{O} 2$ & $1.4437(14)$ & $\mathrm{C} 2-\mathrm{H} 2 \mathrm{~B}$ & 0.9700 \\
\hline $\mathrm{S} 2-\mathrm{O} 3$ & $1.4539(14)$ & $\mathrm{C} 3-\mathrm{C} 4$ & $1.510(2)$ \\
\hline $\mathrm{S} 2-\mathrm{O} 1$ & $1.4606(13)$ & $\mathrm{C} 3-\mathrm{H} 3 \mathrm{~A}$ & 0.9700 \\
\hline $\mathrm{S} 3-\mathrm{S} 4$ & $2.0332(5)$ & C $3-\mathrm{H} 3 \mathrm{~B}$ & 0.9700 \\
\hline $\mathrm{S} 4-\mathrm{O} 4$ & $1.4507(12)$ & $\mathrm{C} 4-\mathrm{H} 4 \mathrm{~A}$ & 0.9700 \\
\hline $\mathrm{S} 4-\mathrm{O} 6$ & $1.4546(13)$ & $\mathrm{C} 4-\mathrm{H} 4 \mathrm{~B}$ & 0.9700 \\
\hline $\mathrm{S} 4-\mathrm{O} 5$ & $1.4623(13)$ & $\mathrm{C} 5-\mathrm{C} 6$ & $1.518(2)$ \\
\hline $\mathrm{N} 1-\mathrm{C} 1$ & 1.487 (2) & $\mathrm{C} 5-\mathrm{H} 5 \mathrm{~A}$ & 0.9700 \\
\hline $\mathrm{N} 1-\mathrm{C} 4$ & 1.4876 (19) & $\mathrm{C} 5-\mathrm{H} 5 \mathrm{~B}$ & 0.9700 \\
\hline $\mathrm{N} 1-\mathrm{H} 1$ & $0.83(2)$ & C6-H6A & 0.9700 \\
\hline $\mathrm{N} 2-\mathrm{C} 2$ & $1.485(2)$ & C6-H6B & 0.9700 \\
\hline $\mathrm{N} 2-\mathrm{C} 3$ & 1.489 (2) & $\mathrm{C} 7-\mathrm{C} 8$ & $1.511(2)$ \\
\hline $\mathrm{N} 2-\mathrm{H} 2 \mathrm{AN}$ & $0.95(2)$ & $\mathrm{C} 7-\mathrm{H} 7 \mathrm{~A}$ & 0.9700 \\
\hline $\mathrm{N} 2-\mathrm{H} 2 \mathrm{BN}$ & $0.93(2)$ & $\mathrm{C} 7-\mathrm{H} 7 \mathrm{~B}$ & 0.9700 \\
\hline $\mathrm{N} 3-\mathrm{C} 5$ & 1.4779 (19) & $\mathrm{C} 8-\mathrm{H} 8 \mathrm{~A}$ & 0.9700 \\
\hline $\mathrm{N} 3-\mathrm{C} 8$ & $1.4820(18)$ & $\mathrm{C} 8-\mathrm{H} 8 \mathrm{~B}$ & 0.9700 \\
\hline $\mathrm{N} 3-\mathrm{H} 3$ & $0.837(18)$ & $\mathrm{O} 10-\mathrm{H} 10 \mathrm{~A}$ & $0.85(3)$ \\
\hline $\mathrm{N} 4-\mathrm{C} 6$ & $1.484(2)$ & $\mathrm{O} 10-\mathrm{H} 10 \mathrm{~B}$ & $0.76(3)$ \\
\hline $\mathrm{N} 4-\mathrm{C} 7$ & $1.491(2)$ & $\mathrm{O} 20-\mathrm{H} 20 \mathrm{~A}$ & $0.81(3)$ \\
\hline $\mathrm{N} 4-\mathrm{H} 4 \mathrm{AN}$ & $0.87(2)$ & $\mathrm{O} 20-\mathrm{H} 20 \mathrm{~B}$ & $0.74(3)$ \\
\hline $\mathrm{N} 3-\mathrm{Zn} 1-\mathrm{N} 1$ & $105.78(5)$ & $\mathrm{N} 1-\mathrm{C} 1-\mathrm{H} 1 \mathrm{~B}$ & 108.9 \\
\hline $\mathrm{N} 3-\mathrm{Zn} 1-\mathrm{S} 1$ & $110.79(3)$ & $\mathrm{C} 2-\mathrm{C} 1-\mathrm{H} 1 \mathrm{~B}$ & 108.9 \\
\hline $\mathrm{N} 1-\mathrm{Zn} 1-\mathrm{S} 1$ & $112.90(4)$ & $\mathrm{H} 1 \mathrm{~A}-\mathrm{C} 1-\mathrm{H} 1 \mathrm{~B}$ & 107.7 \\
\hline $\mathrm{N} 3-\mathrm{Zn} 1-\mathrm{S} 3$ & $101.24(4)$ & $\mathrm{N} 2-\mathrm{C} 2-\mathrm{C} 1$ & $109.22(14)$ \\
\hline $\mathrm{N} 1-\mathrm{Zn} 1-\mathrm{S} 3$ & $108.21(4)$ & $\mathrm{N} 2-\mathrm{C} 2-\mathrm{H} 2 \mathrm{~A}$ & 109.8 \\
\hline $\mathrm{S} 1-\mathrm{Zn} 1-\mathrm{S} 3$ & $116.788(16)$ & $\mathrm{C} 1-\mathrm{C} 2-\mathrm{H} 2 \mathrm{~A}$ & 109.8 \\
\hline $\mathrm{S} 2-\mathrm{S} 1-\mathrm{Zn} 1$ & 98.167 (18) & $\mathrm{N} 2-\mathrm{C} 2-\mathrm{H} 2 \mathrm{~B}$ & 109.8 \\
\hline $\mathrm{O} 2-\mathrm{S} 2-\mathrm{O} 3$ & $112.85(10)$ & $\mathrm{C} 1-\mathrm{C} 2-\mathrm{H} 2 \mathrm{~B}$ & 109.8 \\
\hline $\mathrm{O} 2-\mathrm{S} 2-\mathrm{O} 1$ & $110.61(9)$ & $\mathrm{H} 2 \mathrm{~A}-\mathrm{C} 2-\mathrm{H} 2 \mathrm{~B}$ & 108.3 \\
\hline $\mathrm{O} 3-\mathrm{S} 2-\mathrm{O} 1$ & $111.04(10)$ & $\mathrm{N} 2-\mathrm{C} 3-\mathrm{C} 4$ & $109.77(14)$ \\
\hline $\mathrm{O} 2-\mathrm{S} 2-\mathrm{S} 1$ & $109.71(6)$ & $\mathrm{N} 2-\mathrm{C} 3-\mathrm{H} 3 \mathrm{~A}$ & 109.7 \\
\hline $\mathrm{O} 3-\mathrm{S} 2-\mathrm{S} 1$ & $107.00(6)$ & $\mathrm{C} 4-\mathrm{C} 3-\mathrm{H} 3 \mathrm{~A}$ & 109.7 \\
\hline $\mathrm{O} 1-\mathrm{S} 2-\mathrm{S} 1$ & $105.27(6)$ & $\mathrm{N} 2-\mathrm{C} 3-\mathrm{H} 3 \mathrm{~B}$ & 109.7 \\
\hline $\mathrm{S} 4-\mathrm{S} 3-\mathrm{Zn} 1$ & $101.05(2)$ & $\mathrm{C} 4-\mathrm{C} 3-\mathrm{H} 3 \mathrm{~B}$ & 109.7 \\
\hline $\mathrm{O} 4-\mathrm{S} 4-\mathrm{O} 6$ & $110.47(8)$ & $\mathrm{H} 3 \mathrm{~A}-\mathrm{C} 3-\mathrm{H} 3 \mathrm{~B}$ & 108.2 \\
\hline $\mathrm{O} 4-\mathrm{S} 4-\mathrm{O} 5$ & $111.17(9)$ & $\mathrm{N} 1-\mathrm{C} 4-\mathrm{C} 3$ & $113.09(14)$ \\
\hline $\mathrm{O} 6-\mathrm{S} 4-\mathrm{O} 5$ & $112.02(9)$ & $\mathrm{N} 1-\mathrm{C} 4-\mathrm{H} 4 \mathrm{~A}$ & 109.0 \\
\hline $\mathrm{O} 4-\mathrm{S} 4-\mathrm{S} 3$ & $110.45(6)$ & $\mathrm{C} 3-\mathrm{C} 4-\mathrm{H} 4 \mathrm{~A}$ & 109.0 \\
\hline $\mathrm{O} 6-\mathrm{S} 4-\mathrm{S} 3$ & $108.00(6)$ & $\mathrm{N} 1-\mathrm{C} 4-\mathrm{H} 4 \mathrm{~B}$ & 109.0 \\
\hline $\mathrm{O} 5-\mathrm{S} 4-\mathrm{S} 3$ & $104.53(5)$ & $\mathrm{C} 3-\mathrm{C} 4-\mathrm{H} 4 \mathrm{~B}$ & 109.0 \\
\hline
\end{tabular}




$\begin{array}{ll}\mathrm{C} 1-\mathrm{N} 1-\mathrm{C} 4 & 110.39(12) \\ \mathrm{C} 1-\mathrm{N} 1-\mathrm{Zn} 1 & 112.60(10) \\ \mathrm{C} 4-\mathrm{N} 1-\mathrm{Zn} 1 & 109.65(9) \\ \mathrm{C} 1-\mathrm{N} 1-\mathrm{H} 1 & 105.4(13) \\ \mathrm{C} 4-\mathrm{N} 1-\mathrm{H} 1 & 112.1(13) \\ \mathrm{Zn} 1-\mathrm{N} 1-\mathrm{H} 1 & 106.6(13) \\ \mathrm{C} 2-\mathrm{N} 2-\mathrm{C} 3 & 110.50(14) \\ \mathrm{C} 2-\mathrm{N} 2-\mathrm{H} 2 \mathrm{AN} & 111.5(13) \\ \mathrm{C} 3-\mathrm{N} 2-\mathrm{H} 2 \mathrm{AN} & 107.0(14) \\ \mathrm{C} 2-\mathrm{N} 2-\mathrm{H} 2 \mathrm{BN} & 107.5(14) \\ \mathrm{C} 3-\mathrm{N} 2-\mathrm{H} 2 \mathrm{BN} & 114.4(14) \\ \mathrm{H} 2 \mathrm{AN}-\mathrm{N} 2-\mathrm{H} 2 \mathrm{BN} & 106.1(19) \\ \mathrm{C} 5-\mathrm{N} 3-\mathrm{C} 8 & 110.20(12) \\ \mathrm{C} 5-\mathrm{N} 3-\mathrm{Zn} 1 & 112.71(9) \\ \mathrm{C} 8-\mathrm{N} 3-\mathrm{Zn} 1 & 111.95(9) \\ \mathrm{C} 5-\mathrm{N} 3-\mathrm{H} 3 & 109.2(12) \\ \mathrm{C} 8-\mathrm{N} 3-\mathrm{H} 3 & 106.3(12) \\ \mathrm{Zn} 1-\mathrm{N} 3-\mathrm{H} 3 & 106.2(12) \\ \mathrm{C} 6-\mathrm{N} 4-\mathrm{C} 7 & 110.40(12) \\ \mathrm{C} 6-\mathrm{N} 4-\mathrm{H} 4 \mathrm{AN} & 113.4(13) \\ \mathrm{C} 7-\mathrm{N} 4-\mathrm{H} 4 \mathrm{AN} & 107.0(13) \\ \mathrm{C} 6-\mathrm{N} 4-\mathrm{H} 4 \mathrm{BN} & 114.3(13) \\ \mathrm{C} 7-\mathrm{N} 4-\mathrm{H} 4 \mathrm{BN} & 106.1(13) \\ \mathrm{H} 4 \mathrm{AN}-\mathrm{N} 4-\mathrm{H} 4 \mathrm{BN} & 105.1(17) \\ \mathrm{N} 1-\mathrm{C} 1-\mathrm{C} 2 & 113.43(15) \\ \mathrm{N} 1-\mathrm{C} 1-\mathrm{H} 1 \mathrm{~A} & 108.9 \\ \mathrm{C} 2-\mathrm{C} 1-\mathrm{H} 1 \mathrm{~A} & 108.9 \\ \mathrm{C} 4-\mathrm{N} 1-\mathrm{C} 1-\mathrm{C} 2 & 52.0(2) \\ \mathrm{Zn} 1-\mathrm{N} 1-\mathrm{C} 1-\mathrm{C} 2 & 174.88(12) \\ \mathrm{C} 3-\mathrm{N} 2-\mathrm{C} 2-\mathrm{C} 1 & 59.1(2) \\ \mathrm{N} 1-\mathrm{C} 1-\mathrm{C} 2-\mathrm{N} 2 & -56.1(2) \\ \mathrm{C} 2-\mathrm{N} 2-\mathrm{C} 3-\mathrm{C} 4 & -59.4(2) \\ \mathrm{C} 1-\mathrm{N} 1-\mathrm{C} 4-\mathrm{C} 3 & -51.76(19) \\ \mathrm{Zn} 1-\mathrm{N} 1-\mathrm{C} 4-\mathrm{C} 3 & -176.37(11) \\ \mathrm{N} 2-\mathrm{C} 3-\mathrm{C} 4-\mathrm{N} 1 & 56.0(2) \\ & \end{array}$

$\mathrm{H} 4 \mathrm{~A}-\mathrm{C} 4-\mathrm{H} 4 \mathrm{~B}$

N3- C5-C6

$\mathrm{N} 3-\mathrm{C} 5-\mathrm{H} 5 \mathrm{~A}$

$\mathrm{C} 6-\mathrm{C} 5-\mathrm{H} 5 \mathrm{~A}$

$\mathrm{N} 3-\mathrm{C} 5-\mathrm{H} 5 \mathrm{~B}$

$\mathrm{C} 6-\mathrm{C} 5-\mathrm{H} 5 \mathrm{~B}$

$\mathrm{H} 5 \mathrm{~A}-\mathrm{C} 5-\mathrm{H} 5 \mathrm{~B}$

$\mathrm{N} 4-\mathrm{C} 6-\mathrm{C} 5$

$\mathrm{N} 4-\mathrm{C} 6-\mathrm{H} 6 \mathrm{~A}$

$\mathrm{C} 5-\mathrm{C} 6-\mathrm{H} 6 \mathrm{~A}$

$\mathrm{N} 4-\mathrm{C} 6-\mathrm{H} 6 \mathrm{~B}$

$\mathrm{C} 5-\mathrm{C} 6-\mathrm{H} 6 \mathrm{~B}$

$\mathrm{H} 6 \mathrm{~A}-\mathrm{C} 6-\mathrm{H} 6 \mathrm{~B}$

$\mathrm{N} 4-\mathrm{C} 7-\mathrm{C} 8$

$\mathrm{N} 4-\mathrm{C} 7-\mathrm{H} 7 \mathrm{~A}$

$\mathrm{C} 8-\mathrm{C} 7-\mathrm{H} 7 \mathrm{~A}$

N4-C7-H7B

$\mathrm{C} 8-\mathrm{C} 7-\mathrm{H} 7 \mathrm{~B}$

$\mathrm{H} 7 \mathrm{~A}-\mathrm{C} 7-\mathrm{H} 7 \mathrm{~B}$

N3-C $8-\mathrm{C} 7$

$\mathrm{N} 3-\mathrm{C} 8-\mathrm{H} 8 \mathrm{~A}$

$\mathrm{C} 7-\mathrm{C} 8-\mathrm{H} 8 \mathrm{~A}$

N3- C $8-\mathrm{H} 8 \mathrm{~B}$

$\mathrm{C} 7-\mathrm{C} 8-\mathrm{H} 8 \mathrm{~B}$

$\mathrm{H} 8 \mathrm{~A}-\mathrm{C} 8-\mathrm{H} 8 \mathrm{~B}$

$\mathrm{H} 10 \mathrm{~A}-\mathrm{O} 10-\mathrm{H} 10 \mathrm{~B}$

$\mathrm{H} 20 \mathrm{~A}-\mathrm{O} 20-\mathrm{H} 20 \mathrm{~B}$

$\mathrm{C} 8-\mathrm{N} 3-\mathrm{C} 5-\mathrm{C} 6$

$\mathrm{Zn} 1-\mathrm{N} 3-\mathrm{C} 5-\mathrm{C} 6$

$\mathrm{C} 7-\mathrm{N} 4-\mathrm{C} 6-\mathrm{C} 5$

N3- C5- C6- N4

$\mathrm{C} 6-\mathrm{N} 4-\mathrm{C} 7-\mathrm{C} 8$

$\mathrm{C} 5-\mathrm{N} 3-\mathrm{C} 8-\mathrm{C} 7$

$\mathrm{Zn} 1-\mathrm{N} 3-\mathrm{C} 8-\mathrm{C} 7$

N4-C7-C8-N3
107.8

113.06 (12)

109.0

109.0

109.0

109.0

107.8

110.10 (13)

109.6

109.6

109.6

109.6

108.2

110.19 (13)

109.6

109.6

109.6

109.6

108.1

$112.29(12)$

109.1

109.1

109.1

109.1

107.9

109 (3)

$100(3)$

$-53.62(19)$

$-179.47(11)$

$-57.01(18)$

55.66 (19)

$58.03(18)$

54.16 (18)

$-179.56(11)$

-56.96 (19)

Hydrogen-bond geometry $\left(A,{ }^{\circ}\right)$

\begin{tabular}{lllll}
\hline$D-\mathrm{H}^{\prime} \cdots A$ & $D-\mathrm{H}$ & $\mathrm{H} \cdots A$ & $D \cdots A$ & $D-\mathrm{H} \cdots A$ \\
\hline $\mathrm{N} 1-\mathrm{H} 1 \cdots \mathrm{O} 2^{\mathrm{i}}$ & $0.83(2)$ & $2.25(2)$ & $3.041(2)$ & $160(2)$ \\
$\mathrm{N} 2-\mathrm{H} 2 A N \cdots \mathrm{O} 6^{\mathrm{ii}}$ & $0.95(3)$ & $1.80(3)$ & $2.730(2)$ & $166(2)$ \\
$\mathrm{N} 2-\mathrm{H} 2 B N \cdots \mathrm{O} 10$ & $0.93(2)$ & $1.89(2)$ & $2.811(3)$ & $170(2)$ \\
$\mathrm{N} 3-\mathrm{H} 3 \cdots \mathrm{O} 3^{\mathrm{i}}$ & $0.84(2)$ & $2.03(2)$ & $2.853(2)$ & $166(2)$ \\
$\mathrm{N} 4-\mathrm{H} 4 A N \cdots \mathrm{O} 20$ & $0.87(2)$ & $2.09(2)$ & $2.892(2)$ & $154(2)$ \\
$\mathrm{N} 4-\mathrm{H} 4 B N \cdots \mathrm{O} 5^{\mathrm{iii}}$ & $0.94(2)$ & $1.84(2)$ & $2.763(2)$ & $169(2)$ \\
$\mathrm{O} 10-\mathrm{H} 10 A \cdots \mathrm{O} 4$ & $0.85(3)$ & $1.94(4)$ & $2.780(2)$ & $175(3)$ \\
$\mathrm{O} 10-\mathrm{H} 10 B \cdots \mathrm{O} 1^{\text {iv }}$ & $0.76(3)$ & $2.02(3)$ & $2.777(2)$ & $176(4)$
\end{tabular}


supporting information

$\begin{array}{lllll}\mathrm{O} 20-\mathrm{H} 20 A \cdots \mathrm{O} 1^{\mathrm{v}} & 0.82(3) & 2.02(3) & 2.804(2) & 163(3) \\ \mathrm{O} 20-\mathrm{H} 20 B \cdots \mathrm{O} 5^{\text {vi }} & 0.74(3) & 2.17(3) & 2.866(2) & 158(3) \\ \mathrm{C} 3-\mathrm{H} 3 A \cdots \mathrm{O} 6^{\mathrm{iv}} & 0.97 & 2.45 & 3.221(2) & 136 \\ \mathrm{C} 4-\mathrm{H} 4 A \cdots \mathrm{O} 3 & 0.97 & 2.49 & 3.175(3) & 128 \\ \mathrm{C} 4-\mathrm{H} 4 B \cdots \mathrm{O} 4 & 0.97 & 2.54 & 3.463(2) & 159 \\ \mathrm{C} 5-\mathrm{H} 5 B \cdots \mathrm{S} 3 & 0.97 & 2.86 & 3.453(2) & 120 \\ \mathrm{C} 8-\mathrm{H} 8 B \cdots \mathrm{O} 2 & 0.97 & 2.50 & 3.318(2) & 142\end{array}$

Symmetry codes: (i) $-x+1,-y+1,-z$; (ii) $x+1, y, z$; (iii) $x, y+1, z$; (iv) $-x+1,-y,-z$; (v) $-x,-y+1,-z$; (vi) $-x,-y+1,-z+1$. 